\title{
Evaluation of the Complexity of Automated Trace Alignment using Novel Power Obfuscation Methods
}

\author{
Bozhi Liu, Kemeng Chen, Minjun Seo, Janet Wang, Roman Lysecky \\ University of Arizona, Tucson, AZ, USA \\ bozhiliu@email.arizona.edu, kemengchen@email.arizona.edu, mjseo@email.arizona.edu, meilingw@email.arizona.edu, \\ rlysecky@ece.arizona.edu
}

\begin{abstract}
This paper presents a methodology for evaluating power obfuscation approaches that seek to obfuscate the location of sensitive operations in the power trace, thereby increasing the complexity of automated trace alignment. The paper presents a new adversary model and proposes a new metric, mean trials to success (MTTS), to evaluate power obfuscation methods in the context of automated trace alignment. We evaluate two common obfuscation methods, namely instruction shuffling and random instruction insertion, and we present a new obfuscation method using power shaping to intentionally mislead the attacker.
\end{abstract}

\section{CCS CONCEPTS}

- Security and privacy Side-channel analysis and countermeasures $\cdot$ Security and privacy $\sim$ Embedded systems security

\section{KEYWORDS}

Power obfuscation, power shaping, automated trace alignment, side channel attacks, embedded systems

\section{ACM Reference format:}

B. Liu, K. Chen, M. Seo, J. Roveda, and R. Lysecky. 2018. Evaluation of the Complexity of Automated Trace Alignment using Novel Power Obfuscation Methods In 2018 Great Lakes Symposium on VLSI, Chicago, IL USA, May 2018 (GLSVLSI'18), 4 pages. https://doi.org/10.1145/ 3194554.3194640

\section{INTRODUCTION}

Embedded systems are being widely used in critical systems across a broad range of applications, and they are often more susceptible to attacks by adversaries trying to gain access to or extract sensitive information from the system. Side channel attacks (SCAs) have been proved to be effective across a broad

Permission to make digital or hard copies of all or part of this work for personal or classroom use is granted without fee provided that copies are not made or distributed for profit or commercial advantage and that copies bear this notice and the full citation on the first page. Copyrights for components of this work owned by others than ACM must be honored. Abstracting with credit is permitted. To copy otherwise, or republish, to post on servers or to redistribute to lists, requires prior specific permission and/or a fee. Request permissions from Permissions@acm.org.

GLSVLSI '18, May 16-18, 2018, Chicago, IL, USA

(C) 2018 Association for Computing Machinery.

ACM ISBN 978-1-4503-5724-1/18/05...\$15.00

https://doi.org/10.1145/3194554.3194640 range of systems in extracting sensitive information. Various kinds of side channels exist, including power, electromagnetic radiation, sound, etc.

Automated trace alignment methods are essential for a practical SCA. An attack requires three key steps: 1) automatically determining the information leakage location in each trace, 2) aligning all collected traces, and 3) performing the SCA. SCAs assume the location of sensitive operations in each raw power trace has been determined and that all power traces have been properly aligned. Limited research has addressed the challenge of obfuscating the location of sensitive operations and increasing the complexity of automatic trace alignment to improve the resilience to SCAs.

In this paper, we develop a new adversary model and mean trials to success (MTTS) metric to evaluate the difficulty of automatically identifying sensitive operations within each power trace for a target system. We present a framework for evaluating the effectiveness of existing power obfuscation methods, namely instruction shuffling and random instruction insertion. We further introduce a novel power obfuscation technique called Power Re-Shaping and Mimicry (PReSM) that hides sensitive operations and intentionally mislead attackers to incorrect locations in a power trace. We evaluate the effectiveness of different power obfuscation methods for hiding the location of the sensitive operations and different correlation methods to calculate the MTTS.

\section{RELATED WORK}

SCAs can be categorized as profiled and non-profiled attacks. In non-profiled attacks, including differential power analysis (DPA) [1], correlation power analysis (CPA) [3], or mutual information analysis (MIA) [4], attackers build power model for each key and use a distinguisher to find the best guess. On the other hand, a profiled attack [2] builds power templates for different keys from power traces on a duplicate device and use the templates to guess the key for new power trace.

SCA countermeasures can be summarized in three categories. The first category decreases the signal to noise ratio of the physical implementation. The second category changes the timing profile of the power traces, such as shuffling [5] and instruction reordering [1], these can be mitigated by preprocessing techniques such as signal integration [6] or better statistical methods to differentiate the traces [7]. The third category, masking [8], adds random masks to vulnerable intermediate operations to change its power profile and reduce 
information leakage. These methods focus primarily on the obfuscated power trace given the assumption that trace alignment can be performed ideally. Misaligned traces make SCAs more difficult and less effective [9]. Different from the above works, this paper focuses on evaluating the effectiveness of power obfuscation methods to hide the location of sensitive operations, which makes aligning power traces significantly more difficult.

Existing frameworks [6] for evaluating SCA attacks and countermeasures can be utilized, and researchers have proposed using different statistical tools like mutual information [10] and QMS [7] combined with success rate analysis [11]. Different from described metrics above, this paper proposes a novel Mean Trials to Success (MTTS) metric to indicate the difficulty of locating sensitive operations in power traces with an attacker model using different correlation based methods.

\section{THREAT MODEL}

We assume the adversary has sufficient knowledge of the target device, including the software executing on device, execution sequences, and knowledge of the targeted sensitive operations. With physical access to the device, the adversary has sufficient information to collect power traces within a few thousand cycles of the sensitive operation. However, for a successful SCA, the power traces must be more precisely aligned, typically within a few cycles [9]. To more precisely align the trace, the adversary may construct a pattern of the sensitive operation and use correlation methods to identify the location of the sensitive operations with each collected power traces. Correlation methods may include cross-correlation, Pearson correlation, and mutual information. Additionally, the adversary is expected to know that power obfuscation is being utilized.

\section{SENSITIVE OPERATION IDENTIFICATION}

The power consumed by the software instruction can be modeled as two parts: the base instruction power and the datadependent switching power. Base instruction power is the power consumed without data-dependent switching activity. The datadependent switching power is caused by the switching of gates and registers within the processor. Minimally, the datadependent switching power is proportional to the number of different bits between the instruction's operands and results.

The first step to perform power analysis is to identify the location of the operation where the sensitive information is present. Since the power consumption of the sensitive operations is a distinguishable pattern, the power consumption profile of the pattern can be used as a power pattern to identify when and where sensitive operations start within the power trace.

Using the base instruction power consumption, a pattern of the power trace for the targeted execution sequence can be constructed. The correlation between the trace pattern and power trace is constructed, which indicates how likely each location of the power trace matches the trace pattern. Three correlation methods are considered to identify the location of the targeted sensitive operation: cross-correlation, Pearson correlation, and mutual information.

\subsection{Cross-correlation}

The cross-correlation correlates the trace pattern with the entire power trace to find the location of the targeted sensitive operations. Cross-correlation is defined as:

$(x \star y)[n]=\sum_{m=-\infty}^{\infty} x^{*}[m] y[m+n]$ (1)

where $x$ is the power trace containing the sensitive operations and $y$ is the trace of interest. Since the trace pattern and power traces are digitized time series, we use discrete cross-correlation. The cross-correlation's amplitude indicates the correlation level between the trace pattern and power trace.

\subsection{Pearson Correlation}

Pearson correlation detects a general linear dependency between time series, calculated as:

$\rho_{X, Y}=\frac{\operatorname{cov}(X, Y)}{\sigma_{X} \sigma_{Y}}$

$\operatorname{cov}(X, Y)$ calculates the covariance between $X$ and $Y . \sigma_{X}$ is the variance of $X$. Pearson correlation is calculated between the trace pattern and power trace at every possible location.

\subsection{Mutual Information}

Mutual information measures the information overlap between two sets of data. Given time series $X$ and $Y$, the mutual information between $X$ and $Y$ is calculated as:

$I(X, Y)=\sum_{y \in Y} \sum_{x \in X} p(x, y) \log \left(\frac{p(x, y)}{p(x) p(y)}\right)$

$p(x, y)$ is the joint probability of variable $x$ and $y \cdot p(x)$ and $p(y)$ are the marginal probability. The joint probability divided by product of the marginal probability of $x$ and $y$ reflects the dependency between $x$ and $y$.

\section{POWER TRACE OBFUSCATION}

The goal of power obfuscation is to obfuscate the power trace such that automatically identifying the sensitive operations' location using state-of-the-art correlation methods is hindered. We evaluate three power obfuscation methods: instruction shuffling (IS), random instruction insertion (RII), and Power ReShaping and Mimicry (PReSM). For each obfuscation method, an obfuscation level is defined to control the amount of obfuscation applied. Fig. 1 presents an example of a single original trace and obfuscated traces applying each power obfuscation method.

\subsection{Instruction Shuffling}

Instruction shuffling (IS) reorders the instruction execution, changing both the timing and power profile of the target system. We implement instruction shuffling stochastically by randomly swapping instructions if no dependencies between those instructions exist. Each instruction in the execution sequence is analyzed to identify data and control dependencies on other instructions. For each pair of instructions with no dependency, 


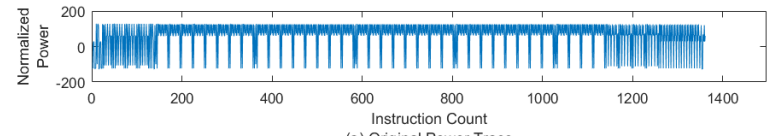

(a) Original Power Trace
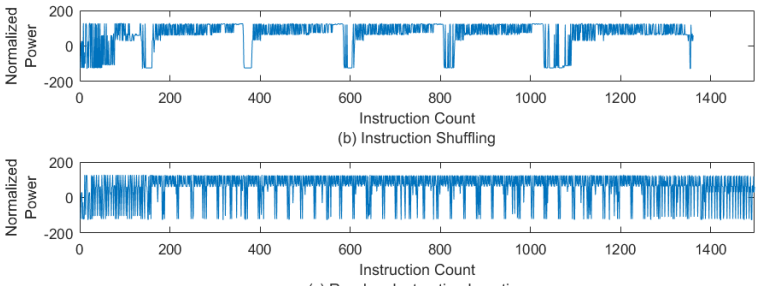

(c) Random Instruction Insertion

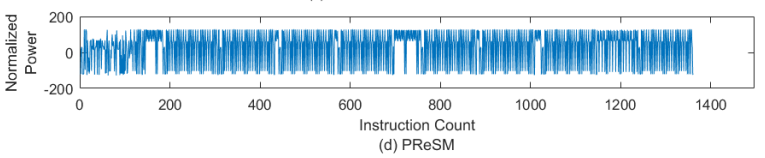

Figure 1: Example actual power traces for target device.

we decide if instructions are shuffled using a shuffling level $L$, which ranges from 0 to 100 . A shuffling level $L$ means that for each possible pair the probability they are swapped is $L \%$.

\subsection{Random Instruction Insertion}

Random instruction insertion (RII) inserts randomly selected instructions with randomly selected operands at random locations in the software execution sequence. The only restriction on the inserted instructions is that the destination register/memory must not overwrite live data. The insertion level defines the percentage, $P$, of new instructions within an instruction sequence to randomly insert.

\subsection{Power Re-Shaping and Mimicry}

Power reshaping and mimicry (PReSM) tries to first reshape the power trace of the sensitive operation so that it appears to be unrelated, and then reshapes the power trace of other operations to mimic the sensitive operation, thereby both hiding the sensitive operation and potentially misleading attackers. For a given raw power trace, the first step is to add additional power to reshape the sensitive operations into a random sequence, which will hide the correct location of sensitive operations from correlation analysis. To mislead the attacker, the next step is to add additional power in several random locations that mimic the sensitive operation (i.e., have a high correlation with the target trace pattern), which will mislead an attacker's automated trace alignment. Finally, to compensate for power trace segments without any additional power, a constant power noise is added.

\section{MEAN TRIALS TO SUCCESS}

MTTS defines the average number of trials an attacker needs before successfully locating the target sensitive operation in a single trace. This metric quantitatively measures the obfuscation effect of different countermeasures for the sensitive operations. Fig. 3 presents an overview of our evaluation methodology for calculating the MTTS for a specific power obfuscation method

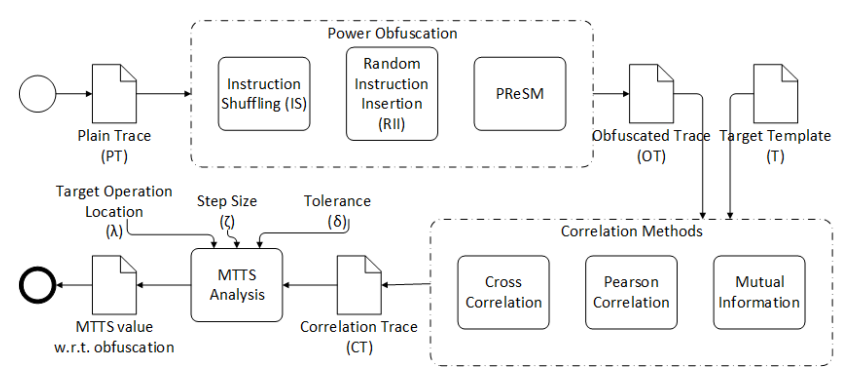

Figure 3: Methodology for calculating the MTTS given a power obfuscation method and correlation method.

ALGORITHM 1: Mean trials to success (MTTS) analysis

Inputs: Correlation Trace $(C T)$, Sensitive Operation Location $(\lambda)$, Step Size $(\zeta)$, Tolerance $(\delta)$

Output: Mean Trials to Success (MTTS)

1. $C T S=\operatorname{sort}(C T)$

2. Bins $=$ DivideIntoBins $($ CTS, ל);

3. $M T T S=0$;

4. for each bin in Bins do

5. for $i=0$ to bin.size do

6. if bin[i].location $\geq(\lambda-\delta)$ and bin.location $\leq(\lambda+\delta)$ then

7. $M T T S+=$ bin.size ${ }^{*} 0.5$

8. return $M T T S$

9. $M T T S+=$ bin.size

10. return $M T T S$

using a specific correlation method. Given an obfuscated plain trace $(P T)$, the selected power obfuscation method is applied to produce the obfuscated trace $(O T)$. Using the trace pattern $(P)$ for the target operation and the selected correlation method, the correlation trace $(C T)$ is calculated. The correlation trace is the input to the MTTS calculation algorithm.

Algorithm 1 presents the MTTS analysis algorithm, which assumes an attacker knows which power obfuscation is used. Rather than simply selecting the location with the highest correlation, the attacker would select a set of $\zeta$ highest correlated locations and analyze those locations in no particular order before looking at the next set of $\zeta$ highest correlated locations. In the MTTS analysis algorithm, $\zeta$ is the step size used to divide a sorted correlation trace (CTS) into bins. Bins are analyzed in descending order, but the locations within each bin can be analyzed in random. The MTTS analysis algorithm additionally has an input for the tolerance $(\delta)$, which defines how close, in cycles, an attacker' guess to the real correct location has to be for a successful attack.

\section{EXPERIMENTAL RESULTS}

MTTS evaluates both the effectiveness of the power obfuscation methods to hide the sensitive operation's location and the effectiveness of the correlation methods as a countermeasure for automated trace alignment. Our target system consists of an ARM processor-based embedded system executing the ARMEmbed AES implementation compiled using arm-linux-gnueabi5. We use a simulation-based approach that combines a cycle accurate gem5 simulation [12] with an instrumented version of McPAT [13] to create cycle-by-cycle power traces of the 


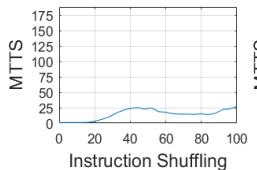

(a)

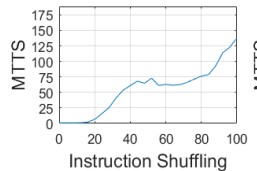

(d)

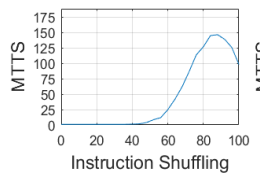

(g)

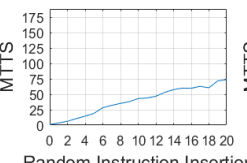

(b)

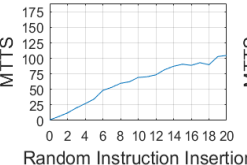

(e)

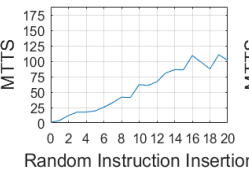

(h)

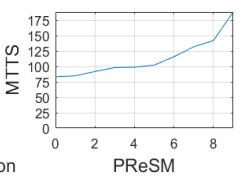

(c)
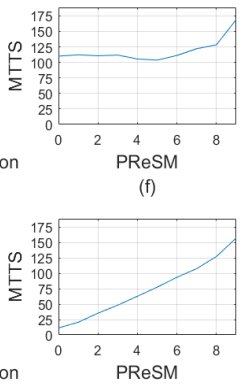

(i)
Figure. 4: MTTS using a single obfuscation method for varying obfuscation levels. $X$-axis represents the obfuscation level for each obfuscation method.

software execution. The first-round AES encryption is the targeted sensitive operation, whereas the entire AES power trace corresponds to the entire process. For performing the MTTS analysis we use a step size $\zeta=1 \%$ and a tolerance $\delta=5$.

Fig. 4 shows the MTTS for each power obfuscation method with increasing obfuscation levels and for each correlation method. Rows represent the correlation method used, namely cross-correlation (top), Pearson correlation (middle), and mutual information (bottom). Columns represent the power obfuscation used, namely instruction shuffling (left), random instruction insertion (middle), and PReSM (right). For each combination of power obfuscation and correlation, we present the average MTTS across 500 executions of the software. Because the experimental power trace corresponds to the AES encryption, which is already isolated from the full system power trace, the presented MTTS values are a conservative estimate of the effectiveness of the obfuscation methods.

IS achieves a best-case MTTS of 25, which is significantly lower than other power obfuscation methods. Notably, the performance of IS saturates, and the best MTTS is not at the highest shuffling level. With a high shuffling level, previous shuffled instructions may be shuffled back closer to their original location, thus counteracting the goal of obfuscation. In contrast, RII exhibits a linearly increase in MTTS with an increase in the obfuscation level, achieving a maximum MTTS of 74 . The linear increase is achieved irrespective of the correlation method used. PReSM achieves the best MTTS of 168 , which is a $6.7 \mathrm{X}$ and $2.3 \mathrm{X}$ increase compared to IS and RII, respectively. Furthermore, PReSM achieves an MTTS equal to the highest MTTS for IS and RII at obfuscation levels of 1 and 5, respectively.

For attackers, knowing the correlation method that yields the lowest MTTS for a specific power obfuscation method provides an advantage for aligning trace. For IS, mutual information is the best correlation method for low obfuscation levels, but crosscorrelation is better when the shuffling level exceeds 60. For a shuffling level of 80 , cross-correlation yields an $84 \%$ lower MTTS. For RII, Pearson correlation and mutual information provide the same performance, but cross-correlation is better at countering this obfuscation method with a maximum decrease in MTTS of $33 \%$. For PReSM, mutual information is the best correlation method across all obfuscation levels, although at highest obfuscation level Pearson correlation's performance is similar (MTTS of 168 for Pearson correlation and 157 for mutual information). To identify the correct locations, cross-correlation and mutual information are the overall best options. Pearson correlation only has comparable performance for RII, but exhibits worse performance in all other cases.

\section{CONSLUSIONS}

We presented a new metric to analyze the difficulty of an attacker to systematically identify the location of sensitive operations in a power trace. The MTTS metric provides designers a new perspective to analyze the effectiveness of power obfuscation method. We further presented a novel power obfuscation approach that reshapes the power trace to intentionally mislead attackers to incorrect locations while hiding the real location. PReSM performs best across the considered power obfuscating methods, with a $2.3 \mathrm{X}$ increase in MTTS compared to the next best obfuscation method.

\section{ACKNOWLEDGMENTS}

This research was supported by the Army Research Office under Grant W911NF-16-1-0130.

\section{REFERENCES}

[1] R. Mayer-Sommer. "Smartly analysing the simplicity and the power of simple power analysis on smartcards." Cryptographic Hardware and Embedded Systems, pp. 78-92, 2000.

[2] S. Chari, J.R. Rao, P. Rohatgi. "Template attacks." International Workshop on Cryptographic Hardware and Embedded Systems, pp. 13-28. Berlin, Heidelberg, 2002

[3] E. Brier, C. Clavier, F. Olivier. "Correlation power analysis with a leakage model." Cryptographic Hardware and Embedded Systems, pp. 16-29, 2004.

[4] B. Gierlichs, L. Batina, P. Tuyls, B. Preneel. "Mutual information analysis." Cryptographic Hardware and Embedded Systems, pp. 426-442. 2008.

[5] C. Herbst, E. Oswald, S. Mangard. "An AES smart card implementation resistant to power analysis attack." International Conference on Applied Cryptography and Network Security, pp. 239-252. 2006.

[6] T.S. Messerges. "Using second-order power analysis to attack DPA resistant software." Cryptographic Hardware and Embedded Systems, pp. 238-251, 2000.

[7] J. Demme, R. Martin, A. Waksman, S. Sethumadhavan. "A Quantitative, Experimental Approach to Measuring Processor Side-Channel Security." IEEE Micro, vol. 33, no. 3, pp. 68-77, May-June 2013.

[8] C. H. Gebotys. "A table masking countermeasure for low-energy secure embedded systems." IEEE Transactions on Very Large Scale Integration (VLSI) Systems, vol. 14, no. 7, pp. 740-753, July 2006.

[9] J.G. Van Woudenberg, M.F. Witteman, B. Bakker. "Improving differential power analysis by elastic alignment." RSA Conference, pp. 104-119, 2011.

[10] L. Batina, B. Gierlichs, E. Prouff, M. Rivain, F.X. Standaert, N. VeyratCharvillon. "Mutual information analysis: a comprehensive study." Journal of Cryptology 24, no. 2, pp 269-291, 2011.

[11] S. Tillich and C. Herbst. "Attacking State-of-the-Art Software Countermeasures-A Case Study for AES," Cryptographic Hardware and Embedded Systems, pp. 228-243. 2008.

[12] N. Binkert et al. "The gem5 simulator." ACM SIGARCH Computer Architecture News 39, no. 2, pp 1-7, 2011.

[13] S. Li et al. "McPAT: an integrated power, area, and timing modeling framework for multicore and manycore architectures." International Symposium on Microarchitecture, pp. 469-480, 2009. 